\title{
A Novel Remaining Useful Life Prediction Framework for Lithium-ion Battery Using Grey Model and Particle Filtering
}

\author{
Lin Chen ${ }^{1} \mid$ Huimin Wang ${ }^{1} \mid$ Jing Chen $^{1} \mid$ Jingjing $\mathrm{An}^{1} \mid$ Bing Ji$^{2} \mid$ Zhiqiang Lyu ${ }^{3}$ | \\ Wenping $\mathrm{Cao}^{4} \mid$ Haihong Pan $^{1}$
}

${ }^{1}$ College of Mechanical Engineering \& Guangxi Key Laboratory of

Electrochemical Energy Materials, Collaborative Innovation Center of

Renewable Energy Materials

(CICREM), Guangxi University,

Nanning, 530000, China

${ }^{2}$ Department of Engineering,

University of Leicester, Leicester,

LE1 7RH, U.K.

${ }^{3}$ State Key Laboratory of Structural Analysis for Industrial Equipment,

Dalian, 116000, China

${ }^{4}$ School of Engineering and Applied

Science, Aston University,

Birmingham, B4 7ET, U.K.

\section{Correspondence}

Haihong Pan, College of Mechanical

Engineering, Guangxi University,

Naning, 530000, China

Email: hustphh@163.com

\section{Present Address}

No.100 Daxue Road, Xixiangtang

District, Nanning City, Guangxi

Province, China

\section{Funding Information}

National Natural Science Foundation of China, Grant/Award Number:

51667006

\begin{abstract}
Summary
An accurate remaining useful life (RUL) prediction method is significant to optimize the lithium-ion batteries' performances in an intelligent battery management system. Since the construction of battery models and the initialization of algorithms require a large amount of data, it is difficult for conventional methods to guarantee the RUL prediction accuracy when the available data are insufficient. To solve this problem, a synergy of slidingwindow grey model (SGM) and particle filter (PF) is exploited to build an innovative framework for battery RUL prediction. The SGM is adopted to explore the modeling of battery capacity degradation, and it characterizes the capacity changes during the battery's life-time with a few data (e.g. 8 data points). To promote the accuracy and traceability of prediction, the development coefficient of the SGM, which can dynamically reflect the capacity degradation, is extracted to update the state variables of state transition function in PF. Accordingly, the fusion of SGM and PF (SGM-PF) can extrapolate the changes of the capacity and realize RUL prediction using fewer data. Furthermore, the performances of SGM-PF are comprehensively validated using two types of batteries aged under different conditions. The RUL prediction results reveal that the SGM-PF framework can achieve precise and reliable predictions in different prediction horizons with as few as 8 data points, and it has prominent performance in accuracy and stability over contrastive methods, especially in long-term prognosis.
\end{abstract}

\section{KEYWORDS}

Lithium-ion battery, remaining useful life, prediction, grey model, particle filter.

\section{INTRODUCTION}

Lithium-ion batteries (LIBs) have been extensively considered as power sources and energy tanks thanks to their high energy density, low self-discharge rate and long lifecycle, and have been applied in various fields, for example electric vehicles, consumer electronics, micro grids and space ships. Nevertheless, a critical issue for LIB is that its performance deviation and degradation will result in unsafety and instability. LIB degradation without maintenance is likely to have a detrimental impact on the performance and reliability of the system and it may cause catastrophic failures. Thus, it is necessary to explore an effective and accurate technique for the remaining useful life (RUL) prediction of LIBs, and accurate RUL prediction plays an imperative role in optimizing battery energy arrangement $[1,2]$. Accurate RUL prediction can provide the available service time and the decision information for timely replacement of degraded batteries before their capacity reaches $80 \%$ of initial capacity, namely, the end of life (EOL) [3].

The current prediction methods of RUL can be broadly divided into two categories: data- 
driven methods and filter-based methods.

The data-driven methods make full use of some machine learning algorithms to automate RUL predictions. They are model-free and independent on models featuring degradationdependent parametric drift. Liu et al. [4] proposed an incremental optimized relevance vector machine (RVM) framework for RUL prediction, which used a novel online training strategy to lessen the computational burden of the kernel function. The RUL prediction results indicated that their framework could generate precise predictions with faster learning speed. Hu et al. [5] utilized the sparse Bayesian predictive modeling (SBPM) methodology to detect the underlying correlation between capacity loss and sample entropy extracted from discharge voltage. Also, the authors performed a combination of SBPM and bootstrap sampling concepts for RUL. The neural networks are also widely used in recent researches. For instance, Wu et al. [6] presented an online approach based on the feed forward neural network and importance sampling to estimate RUL of LIBs. Zhang et al. [7] developed a long short-term memory recurrent neural network (LSTMRNN) method to achieve RUL prediction with lower dependence on data volume than traditional RNNs. Moreover, they utilized the Monte Carlo method to simulate the probability distribution of prediction results. These data-driven methods can realize RUL prediction without conducting intensive study on battery degradation mechanism. However, their initialization depended on the quantity of historical data. Consequently, the prediction results from data-driven methods may not be accurate if the historical data are insufficient or poor in quality.

The first procedure of the filter-based methods was the construction of a nonlinear degradation model from different perspectives and some advanced filter techniques were applied to the prediction process, such as particle filter (PF). Zhang et al. [8] utilized the nonlinear least squares to establish a battery capacity degradation model with an exponential function, and the PF was employed to update the model parameters and predict the RUL. To track the battery's degradation trend more precisely over its life cycle, Xing et al. [9] combined the empirical exponential model with a polynomial regression model and adopted the PF for parameter tracking and RUL prediction. For further improvement in filter techniques, Miao et al. [10] developed an improved PF method utilizing the unscented Kalman filter (UKF) to reconstruct the proposal distribution in PF. The RUL prediction results of their method was proved to be more accurate than those of the PF. To improve the battery model in PF framework, Su et al. [11] applied the interacting multiple model particle filter (IMMPF) to RUL prediction. The IMMPF adopted three improved degradation models to get accurate prediction results. Considering the problem of poor performance caused by model limitations in long-term prognostics, Chang et al. [12] demonstrated a degradation model using UKF-based prognostic error and the prediction results from their study were updated based on the error-correction theory. Generally, the degradation model is constructed in advance based on the existing measurement values, however, sufficient degradation data are required for RUL predictor initialization. The prediction results using filterbased methods are usually influenced by the accuracy of a priori known model. Moreover, it is difficult to derive an accurate analytical model for a complex dynamic LIB system, especially when the system operates under a noisy and unpredicted environment.

In the current study, the authors developed a novel framework for battery RUL prediction, which fused the algorithm of sliding-window grey model (SGM) with the particle filtering (PF), to realize the reliable prognostics with a small amount of data. The SGM was adopted to describe the complex battery degradation throughout the batteries' life-time. The sliding-window 
mechanism of SGM adopts new data and update the model parameters dynamically throughout the battery life cycle, which ensures the traceability of degradation trend by exploring the variation in a few data (as few as 8 sampling points). The development coefficient of the SGM, representing the historical degradation trend, was employed to update the state transition function of PF. The renewed state transition function reflected the recurrence between two adjacent cycles. Consequently, one of the advantages of this framework is that it can reduce the amount of data required for the model initialization and the RUL prediction. Additionally, the updating mode of battery model parameters is improved as the battery degrades. The proposed SGM-PF framework avoids the data requirement for model fitting or offline training in machine learning algorithm, and it is suitable for the situations in which the historical data are barely available or inadequate. This characteristic makes the SGM-PF framework more applicable for vehicle BMS with limited storage and operation ability.

The rest of this study is structured as follows: Section 2 presents the SGM and describes the SGM-PF framework for RUL prediction in detail. Section 3 provides the experimental tests and datasets. The evaluation of the SGM, the RUL prediction results based on SGM-PF framework are demonstrated and discussed in Section 4, and conclusions are given in Section 5.

\section{METHODOLOGY}

\subsection{Sliding-window Grey Model}

Grey system theory deals with uncertainty systems with its superiority that relatively small sample and poor data information is requested to predict the systems' behaviors [13, 14]. GM (1, 1), as the most basic description of grey system, is established for time series prediction by using as few as four sample data points $[15,16]$. The SGM is devised and proposed, which adopts the sliding mechanism to update model based on the latest information and enhance the prediction performance of the GM $(1,1)$. The modeling procedure of SGM is shown in Fig. 1.

FIGURE. 1 Modeling procedure of SGM

Step 1: For instance, a total number of $S$ data points $(S \geq 4)$ were adopted for model initialization, whilst a number of $N$ data points $(N \geq 1)$ are forecasted by $\operatorname{GM}(1,1)$ of a sequential sliding operation. Starting with $x_{(1)}^{(0)}$, establishing $\operatorname{GM}(1,1)$ by using the historical sequence $\mathbf{X}^{(0)}$ can be written as Eq.(1)

$$
\mathbf{X}^{(0)}=\left\{x_{(k)}^{(0)}\right\}_{k=1}^{S}
$$

where $\left\{x_{(k)}^{(0)}\right\}$ is the historical array sequence at $k$ th.

Step 2: The next sequence $x_{(k)}^{(1)}$ (Eq. 3) can be obtained by conducting the so-called first-order accumulating generation operation (1-AGO). The expression of "1-AGO" is written as follows: 


$$
\mathbf{X}^{(1)}=\left\{x_{(k)}^{(1)}\right\}_{k=1}^{S}
$$

where,

$$
x_{(k)}^{(1)}={ }_{i=1}^{k} x_{(i)}^{(0)}, k=1,2, \ldots, S
$$

Step 3: The whitening differential equation, as the description of $\operatorname{GM}(1.1)$, can be established as follows:

$$
\frac{d x_{(k)}^{(1)}}{d k}+a x_{(k)}^{(1)}=b
$$

where $a$ is the development coefficient, which represents the tendency in data development, and $b$ is a constant coefficient. To obtain the essential parameter $a$, the sequence of parameters $\hat{a}=[a, b]^{\mathrm{T}}$ is calculated as follows:

$$
\hat{a}=\left(\mathbf{B}^{\mathrm{T}} \times \mathbf{B}\right)^{-1} \times \mathbf{B}^{\mathrm{T}} \times \mathbf{Y}_{S}
$$

where

$$
\mathbf{B}=\left[\begin{array}{cc}
-0.5 \times\left(x_{(2)}^{(1)}+x_{(1)}^{(1)}\right) & 1 \\
-0.5 \times\left(x_{(3)}^{(1)}+x_{(2)}^{(1)}\right) & 1 \\
\ldots & \ldots \\
-0.5 \times\left(x_{(S)}^{(1)}+x_{(S-1)}^{(1)}\right) & 1
\end{array}\right]
$$

and

$$
\mathbf{Y}_{S}=\left[\begin{array}{llll}
x_{(2)}^{(0)} & x_{(3)}^{(0)} & \cdots & x_{(S)}^{(0)}
\end{array}\right]^{\mathrm{T}}
$$

The predicted value of accumulated sequence can be estimated by solving the whitening differential equation in Eq. (4), the result is expressed as:

$$
\hat{x}_{(k+1)}^{(1)}=\left(x_{(1)}^{(0)}-\frac{b}{a}\right) \times \exp (a k)+\frac{b}{a}, k=1,2, \ldots, S-1
$$

Step 4: The inverse accumulated generating operation is conducted to calculate the predicted value of next data $\hat{x}_{(k+1)}^{(0)}$ :

$$
\hat{x}_{(k+1)}^{(0)}=\hat{x}_{(k+1)}^{(1)}-\hat{x}_{(k)}^{(1)}, k=1,2, \ldots, S-1
$$

where $\hat{x}_{(1)}^{(0)}=x_{(1)}^{(0)}$. Applying Eq. (8) to Eq. (9) to calculate the predicted value of $\hat{x}_{(k+1)}^{(0)}$ and $\hat{x}_{(k)}^{(0)}$, 
the quotient of $\hat{x}_{(k+1)}^{(0)}$ divided by $\hat{x}_{(k)}^{(0)}$ can be expressed as:

$$
\begin{aligned}
& \hat{x}_{(k+1)}^{(0)} / \hat{x}_{(k)}^{(0)} \\
& =\left(\hat{x}_{(k+1)}^{(1)}-\hat{x}_{(k)}^{(1)}\right) /\left(\hat{x}_{(k)}^{(1)}-\hat{x}_{(k-1)}^{(1)}\right) \\
& =\frac{[1-\exp (a)]\left[x_{(1)}^{(0)}-\frac{b}{a}\right] \exp (-a k)}{[1-\exp (a)]\left[x_{(1)}^{(0)}-\frac{b}{a}\right] \exp (-a(k-1))} \\
& =\exp (-a)
\end{aligned}
$$

The recursive relation of GM is then obtained by changing the form of Eq. (10):

$$
\hat{x}_{(k+1)}^{(0)}=\hat{x}_{(k)}^{(0)} \times \exp (-a)
$$

and subsequent data $\left\{\hat{x}_{(S+1)}^{(0)}, \hat{x}_{(S+2)}^{(0)}, \ldots, \hat{x}_{(S+N)}^{(0)}\right\}$ are predicted by:

$$
\operatorname{GM}(1,1)\left\{x_{(k)}^{(0)}\right\}_{k=1}^{S}\left\{x_{(k)}^{(0)}\right\}_{k=S+1}^{S+N}
$$

Step 5: The main objective of applying the "sliding-window" concept here is to obtain a new development coefficient $a$. Therefore, the GM $(1,1)$ is reconstructed when new data points are obtained. That is, if new data $\left\{x_{(k)}^{(0)}\right\}_{k=S+1}^{S+m}$ are obtained, previous data $\left\{x_{(k)}^{(0)}\right\}_{k=1}^{m}$ will be eliminated and a new GM $(1,1)$ will be constructed based on the sequences $\left\{x_{(k)}^{(0)}\right\}_{k=S+1}^{S+m}$ ( $m$ is the number of new data). The new GM then calculates a new development coefficient.

Step 6: Steps 1 to 5 should be executed repeatedly. When the prediction of the last data point is finished, the process of GM ends.

\subsection{SGM-PF}

The particle filtering (PF) belongs to a special version of Bayes filter, and it obtains the posterior probability density function (PDF) based on sequential Monte Carlo sampling [17]. A group of "particles" from the unknown state space is used to estimate a state PDF by PF, and their weights denote the discrete probability masses. The PF assumes that the state equations can be demonstrated as a first-order Markov process with the independent outputs being conditional. The dynamic process, which is described by the state-space model as shown in Eq. (13):

$$
\left\{\begin{array}{l}
x_{k}=f\left(x_{k-1}, v_{k-1}\right) \\
y_{k}=h\left(x_{k}, w_{k}\right)
\end{array}\right.
$$

where $x_{k}, y_{k}, v_{k}$, and $w_{k}$ denote the state, the corresponding measurement, the process noise and the measurement noise at time $k$, respectively. The process observations are derived from a marginal distribution $p\left\{y_{k} \mid x_{k}\right\}$. Further, $\left\{y_{k}\right\}$ is conditionally independent of all other states. The 
current system state $\left\{x_{k}\right\}$ can be evaluated by calculating the posterior PDF $p\left\{x_{k} \mid y_{1: k}\right\}$.

The main objective of the particle filtering is to approximate and represent the posterior PDF by employing a group of particles $\left\{x_{k}{ }^{(i)}\right\}_{i=1}^{N}$ along with their associated weights $\left\{\omega_{k}^{(i)}\right\}_{i=1}^{N}$. The posterior PDF at $k$ is expressed as follows:

$$
p\left(x_{k} \mid y_{1: k}\right)=\sum_{i=1}^{N} \omega_{k}^{i} \delta\left(x_{k}-x_{k}^{i}\right)
$$

where $\delta(\cdot)$ denotes the function of Dirac delta and $N$ is the number of random samples. $\omega_{k}^{i}$ is the important weight of the particle. The procedure of PF can be referred to [17].

To enhance the traceability of model during the long-term degradation process, it is necessary to employ a dynamic model with low computational cost for continuous modeling in PF algorithm. Thus, an SGM-PF framework was constructed by fusing SGM and PF algorithm. The SGM was adopted to establish a degradation model dynamically for mining the degradation trend. The development coefficient $a$ of SGM was used to promote the state transition function in PF. The SGM-PF framework for RUL prediction of the battery is schematically depicted in FIGURE. 2.

FIGURE. 2 The SGM-based PF framework for RUL prediction

The specific implementation process is as follows:

Step 1: Extracting the battery capacity data by online coulomb counting and removing the outlier data to obtain the historical sequence $\mathbf{X}^{(0)}$, then the SGM can be established with the data using Eqs. (1)-(9);

Step 2: According to Eq. (11), the recursive relation of GM can define the relationship between two capacity values from two adjacent cycles. This relation is formulated as a state transition function between cycle $k$ and cycle $k-1$, with the influence of noises taking into account:

$$
\left\{\begin{array}{l}
x_{k}=x_{k} \exp \left(-a_{k-1}\right)+v_{k} \\
y_{k}=x_{k}+w_{k}
\end{array}\right.
$$

where $x_{k}$ and $y_{k}$ denote the capacities predicted and measured at cycle $k$, respectively. $a_{k-1}$ is the development coefficient at cycle $k-1 ; v_{k}$ and $w_{k}$ represents the process noise and the measurement noise, respectively;

Step 3: Tracking of the change of capacity state $x_{k}$ by PF. The $y_{k}$ is used to update the important weight by the marginal distribution $p\left\{y_{k} \mid x_{k}\right\}$.

Step 4: At cycle $k$, the predicted capacity $x_{k}$ is used as input data of SGM, and the SGM 
output the corresponding development coefficient $a_{k}$ by Eqs. (4)-(7). Then $a_{k}$ is returned to the Step 2 to participate in the prediction at next cycle. Steps (2)-(4) are continuously repeated if the predicted capacity does not reach the pre-defined failure threshold ( $80 \%$ of initial capacity). When the failure threshold is hit, the prediction procedure goes to Step 5.

Step 5: The posterior PDF at cycle $k$ can be estimated by:

$$
p\left(R U L \mid y_{1: k}\right) \approx \sum_{i=1}^{N} \omega_{k}^{i} \delta\left(R U L_{k}-R U L_{k}^{i}\right)
$$

The prediction value of RUL is estimated by:

$$
R \hat{U} L_{k}=\sum_{i=1}^{N} R U L_{k}^{i} \omega_{k}^{i}
$$

The RUL is the number of aging cycles before battery hits the failure threshold. As time proceeds, the RUL estimation results are updated with the new measurements collected.

\section{EXPERIMENTAL TESTS}

Experimental data from two types of LIBs (see Table 1) were selected for cycle-life testing, one was from the NASA datasets, and the other was collected from a test bench using the Samsung ICR 18650 batteries.

Table 1 A comparison of the specifications of two types of batteries

1) The constant current (CC) aging tests: the data were directly compiled from the Prognostics Center of Excellence (PCoE) of the NASA AMES [18]. The samples B05, B06, and B07 ran through in a standard CC - CV charging. Their discharging was conducted by a current of $2 \mathrm{~A}$ until their voltage reached $2.7 \mathrm{~V}, 2.5 \mathrm{~V}$ and $2.2 \mathrm{~V}$ for the samples, respectively. The cyclelife testing was continuously conducted until the end-of-life (EOL) criterion was reached, which was designated as a $30 \%$ fade in the rated capacity of Type 1 (from $2 \mathrm{Ah}$ to $1.4 \mathrm{Ah}$ ).

2) The dynamic current aging tests: the dynamic tests were carried out on the test rig designed by the authors, which can be found in our early works $[19,20]$. A series of tests were conducted to produce more battery degradation data for RUL prediction as a further supplement of NASA battery dataset. The test rig consisted of an environmental chamber, an electronic load instrument (ITECH IT8511A+), a charger (ITECH IT6523D), some customized switching circuits, and a host computer. The failure threshold of the samples No. 17 (a new battery) and No. 30 (a used battery with maximum available capacity of $2.47 \mathrm{Ah}$ ) were defined as $80 \%$ of the capacity. The capacity of No.17 is tested under $0.52 \mathrm{~A} \mathrm{CC}$ discharging at $25^{\circ} \mathrm{C}$. The aging tests for No.17 are conducted at $45^{\circ} \mathrm{C}$, and the discharge current of aging tests is demonstrated as Fig. 3 (a). For No. 30, all the tests are conducted at $35^{\circ} \mathrm{C}$. Its capacity test was under $0.26 \mathrm{~A} \mathrm{CC}$ discharging and aging discharge current is shown in Fig. 3 (b). 
FIGURE. 3 Dynamic-current aging cycle test. (a) Urban Dynamometer Driving Schedule (UDDS) and Pulse Discharge. (b) Pulse Discharge

\section{RESULTS AND DISCUSSION}

\subsection{Degradation Model Analysis of SGM}

Establishing a high-fidelity capacity degradation model of LIBs is a critical premise of precise RUL prediction. The SGM is employed to build the degradation model and estimate the capacity changes throughout the battery cycle life. For each battery sample, eight data points $(S=8)$ are used for sliding-window to build the SGM. Subsequently a new SGM is established with the oldest data point eliminated and one new data point adopted $(m=1)$. Then the new SGM is used to generate the next data point $(N=1)$. The whole process repeats until batteries achieve its EOL, and the capacity prediction results together with the trajectory of development coefficient are shown in FIGURE. 4.

FIGURE. 4 The capacity prediction results by SGM and the trajectories of development coefficient for B05, B06 and B07

The development coefficient $a$ represents the development trend of historical sequence. Thus, we characterize the capacity fading trend using the variation of development coefficient $a$ of SGM. The capacity decreases in a nonlinear manner, and when the capacity fluctuation occurs, the development coefficient has large change correspondingly, such as at approximately the 20th cycle, 30th cycle and 50th cycle. Furthermore, the accuracy of capacity prediction by SGM was validated by comparisons via several evaluation criteria in Table 2, including maximum error (Max error), mean absolute percentage error (MAPE), and standard deviation (STD). The results indicate that the SGM has sufficient accuracy in prediction, irrespective of the capacity trajectory changes for smooth or wavy data series. Evidently, the SGM is able to describe the changing process of capacity degradation effectively.

Table 2 The prediction results of capacity by SGM

To further evaluate the performance of SGM, the battery capacity fading is evaluated by comparing with a traditional second-order polynomial model as a benchmark [9]. The model was empirically established through fitting the different data points for battery degradation modeling initialization (DMI). The results in FIGURE. 5 illustrate the comparative results of capacity prediction by SGM and polynomial model built with different DMI. It can be seen that both methods have good consistency with the raw data for the known capacity trajectory but the prediction results are different. The capacity predicted by the polynomial model has a larger deviation than that predicted by the SGM, which indicates the SGM can achieve higher accuracy. The quantitative statistical analysis for error of capacity prediction for polynomial model and SGM are shown in FIGURE. 6, in which the statistical errors of SGM are all obviously less than 
those of polynomial model. These results illustrate the SGM can achieve accurate prediction and track the capacity development trend. The SGM can be regarded as an effective degradation model for its good traceability and effectiveness of prediction.

FIGURE. 5 The capacity prediction based on polynomial model and SGM at different DMI: (a) B05, (b) B06, and (c) B07

FIGURE. 6 The comparison of capacity prediction error of polynomial model and SGM: (a) Max errors; (b) MAPE; and (c) STD

\subsection{Comparison of RUL Prediction Results}

In this section, the traditional polynomial model-based PF (PPF) and the SGM-PF (SGPF) are compared for RUL prediction. The used data sets are from two types of aging tests (CC and dynamic-current discharging). Herein, we use approximately $1 / 3,1 / 2$, and $2 / 3$ of the original data of battery whole life cycle numbers (WLCN) as the DMI and predict the RUL for validating the performance of the two methods. For the SGM-PF framework in RUL prediction, SGM adopts the last 8 historical data points in the DMI as modeling dataset $(S=8)$. After modeling and PF prediction, the SGM abandons the first data $(m=1)$ from the modeling data, adds the predicted data and continues to calculate the next development coefficient for the PF update. This prediction-and-updating cycle repeats until the predicted capacity reaches the failure threshold.

To validate the practicability of the SGPF framework, the RUL predictions are conducted under different discharging conditions and degree of aging. The RUL prediction results for the two types of batteries under different experimental conditions are shown in FIGURE. 7 and FIGURE. 8. For CC discharge condition, FIGURE. 7 demonstrates the RUL prediction results of B05 and B06 at different DMI (the first 60 cycles, 80 cycles and 100 cycles, respectively). Specifically, for B05 using modeling data from three DMI (FIGURE. 7a-c), the RUL prediction results of SGPF are much closer to the actual EOL than those of PPF. Moreover, the RUL prediction results of SGPF are generally better than those of PPF for both B05 and B06 when the data for modeling are from the same DMI, such as the SGPF prediction error from the DMI of 100 cycles is visibly less than that of PPF (FIGURE. 7c and f). Experimental results indicate that the SGPF have better accuracy than PPF in CC discharge condition with different batteries and using different data for modeling.

FIGURE. 7 RUL prediction for B05 and B06 using PPF and SGPF with different DMI

FIGURE. 8 RUL prediction for No. 17 and No. 30 using PPF and SGPF with different DMI

Fig. 8 demonstrates that the RUL prediction curves of the two Samsung ICR18650 batteries 
at different dynamic-current discharge conditions and with different temperatures. No.17 and No.30 have dissimilar degree of aging, and their experimental temperatures are distinct (as described in Section 3). It is obvious that WLCN of brand-new No.17 is larger than that of No.30, therefore, the DMI for the No.17 are set as 300, 400 and 500 cycles while the corresponding values of No.30 are 70, 100, and 130 cycles. Similar to the NASA batteries (Fig. 7), the SGPF performs better than the PPF does in degradation trajectory tracking and RUL prediction. For example, when the degradation trajectories of both No.17 and No.30 are relatively smooth, the predicted trajectories of SGPF and PPF are both close to their real trajectories, however, the trajectories of SGPF fit the real trajectories better than those of PPF do (Fig. 8a-f). Unfortunately, once violent fluctuations occur near the prediction starting point, the predicted trajectory of PPF deviates dramatically from the real trajectory and causes significant prediction errors, but the SGPF still can achieve accurate long-term prediction results (Fig. 8d). The results of both methods become more precise and credible as the DMI increases, but the SGPF still keeps its superiority in degradation trajectory fitting and prediction accuracy over PPF (Fig. 8d-f).

Overall, the RUL prediction results of SGPF are better than those of PPF. For PPF, insufficient historical data used for modeling may lead to an inaccurate establishment of battery model. Compared to PPF, the SGPF framework possesses these features: 1) the battery model is built using limited historical data, and 2) the model updating mechanism is efficient and it can dynamically adjust the parameters of battery model with the change of battery degradation. That is, the SGPF provides a significant improvement over the PPF, the SGPF framework is immune to different discharge mode in the aging cycle. Also, it keeps stable performance in different prediction horizons. Consequently, the SGPF achieves a higher prediction accuracy in both the $\mathrm{CC}$ and dynamic-current discharging than the PPF does. For computational complexity, SGPF gains a time complexity of $\mathrm{O}\left(n^{2}\right)$, which is the same as that of the PPF, indicating that the SGPF algorithm has the same level of computational processes for data as the PPF does. But the processed data of the SGPF are much fewer than that of PPF, thus, the computational burden of SGPF is relatively lower.

To further verify the superiority of SGPF over traditional methods, a back propagation neural network (BPNN) was applied to compare with the SGPF. B05 and No.17 were selected from each of the two types of LIBs to validate the two methods at different discharge conditions with different DMI (the DMI of the two batteries are the same as above). In Fig.9 (a)-(c), the prediction trajectories using SGPF are almost coincided with real data trajectories before the 125 cycles of B05, while the forecast values deviated from the real data after 125 cycles. However, using SGPF, the prediction trajectories of No.17 can keep good traceability with real trajectories during the entire prediction process (Fig. 9d-f). As for the BPNN, the prediction trajectories of both B05 and No.17 have significant deviations from the real data, especially when the DMI is approximately $1 / 3$ of the WLCN (Fig. 9d). Although the predicted trajectories of BPNN are converging to the real data with the growth of the DMI, the SGPF exhibits better accuracy and stability than BPNN does.

To further justify the superiority of SGPF over common methods, a traditional back propagation neural network (BPNN) was used to compare with the SGPF. B05 and No.17 were chose from each of the two types of LIBs to validate the two methods at different discharge condition with different DMI (the DMI of the two batteries are same as above). In FIGURE.9 (a)(c), the prediction trajectories using SGPF are almost coincided with real data trajectories before 
the 125 cycles of B05, but the forecast values deviated from the real data after 125 cycles. However, the prediction trajectories of No.17 using SGPF keep good traceability with real trajectories all the time (FIGURE. 9d-f). As for the BPNN, the prediction trajectories of both B05 and No.17 have a significant deviation from the real data, especially when the DMI is approximately $1 / 3$ of the WLCN (FIGURE. 9d). Although the prediction trajectories of BPNN are converging to the real data with the DMI increasing, their accuracy and stability are not able to catch up with those of SGPF.

FIGURE. 9 RUL prediction for B05 and No.17 using BPNN and SGPF with different DMI

The $95 \%$ confidence interval (95\% CI), absolute error (AE), root mean square error (RMSE) and an accuracy indicator (AI) are regard as evaluation criteria for quantitative assessing the accuracy of prediction results.

i) $95 \% \mathrm{CI}$ :

A 95\% CI gives an estimated range of predicted RUL, it covers 95\% of the PDF curve and the probability of observing a predicted value outside of this area is less than $5 \%$. Since the BPNN does not involve statistical inferences, the $95 \% \mathrm{CI}$ of its prediction results are not provided.

ii) Absolute error:

$$
\mathrm{AE}=\left|R U L_{\text {real }}-R U L_{\text {predicted }}\right|
$$

where $R U L_{\text {real }}$ is the real RUL, $R U L_{\text {predicted }}$ is the predicted RUL.

iii) Root-mean-square error: a frequently used measure of the differences between values predicted by a model or an estimator and the values actually observed.

$$
\mathrm{RMSE}=\sqrt{\frac{1}{n} \sum_{j=1}^{n}\left(x_{\text {real }}-x_{\text {predicted }}\right)^{2}}
$$

where $x_{\text {actual }}$ and $x_{\text {prediction }}$ are real capacity and predicted capacity values, respectively.

iv) Accuracy indicator: a defined indicator to evaluate the relative accuracy.

$$
\mathrm{AI}=\left(1-\frac{\mathrm{AE}}{R U L_{\text {predicted }}}\right) \times 100 \%
$$

The comparisons of the SGPF, PPF and BPNN on the evaluation criteria are presented in Table 3. Firstly, the impacts of the amount of historical data are proposed. BPNN and PPF use all historical data before DMI, while SGPF solely adopts the newest eight data points for modeling. The results show that SGPF can achieve higher accuracy with less historical data than the other two methods, no matter how the DMI is chosen. Secondly, the impacts of the prediction horizon are revealed. When all five batteries are predicted with small DMI (approximately $1 / 3$ of WLCN), 
the prediction accuracy and stability for all the three methods are relatively poor. For BPNN and PPF, this phenomenon occurs because insufficient historical data used for modeling decrease the degree of regression and fitting, which results in a less accurate establishment of the model and an increase of estimation errors. As for SGPF, the errors are mainly from the cumulative process happened in the repeated modeling in the long-term prediction. However, owing to the model updating mechanism of SGM, these cumulative errors are weakened, and the accuracy of SGPF is still satisfactory and significantly better than that of BPNN and PPF in long-term prediction. When the prediction horizons decrease or the historical data increase, the accuracy of BPNN and PPF becomes obviously higher, but the prediction performance of SGPF is still better than that of BPNN or PPF. Finally, the extensive applicability of the SGPF is also validated. The used batteries come from different manufacturers and they are tested at various conditions, including $\mathrm{CC}$ and dynamic-current discharge at different temperatures. Moreover, the initial aging degree of batteries is not the same. On these premises, the results of SGPF have better performance in all five batteries than those of the other two methods, indicating better applicability of SGPF under various conditions.

Table 3 Prediction results of three methods for five batteries with different DMI

\section{CONLUSIONS AND FUTURE WORK}

In this paper, an innovative framework for battery RUL prediction is synthesized by SGM and PF. The SGM in the proposed framework is used to evaluate the capacity degradation throughout the batteries' life time, and it requires only eight data points for modeling and updating. The effectiveness of SGM is validated via capacity predictions for NASA batteries, and the maximum capacity error of the SGM is solely $0.1039 \mathrm{Ah}$, indicating that the SGM is more precise than the traditional polynomial model. Therefore, the SGM can continuously and effectively update model parameters to reflect the change trend of capacity. Furthermore, the proposed framework adopts the development coefficient of SGM to update the state variables in the state transition function of PF, and the ability of PF in tracking capacity degradation is completely utilized to track the capacity changes and generate RUL predictions. The RUL prediction results highlight the good performance of SGM-PF framework in predicting two types of batteries aged under different aging conditions. Especially, the maximum RUL prediction error for SGM-PF is only 14 cycles, even with eight data points for initialization. Compared with the traditional polynomial model-based PF and the back propagation neural network, the SGM-PF gains higher RUL prediction accuracy with different prediction horizons, and it has prominently better performance in the long-term RUL prediction.

In practical applications, the ambient temperature varies dynamically throughout the batteries' life-time. This research has considered several constant aging temperatures (e.g., $25^{\circ} \mathrm{C}$, $35^{\circ} \mathrm{C}$ and $45^{\circ} \mathrm{C}$ ). In the future research, more experiments will be conducted under changing ambient temperatures to further explore the impacts of temperature on the RUL prediction. Moreover, the future study will expand the implement of SGM-PF to more conditions including depth-of-discharge and energy recovery. 


\section{ACKNOWLEDGEMENTS}

This work was supported by the National Natural Science Foundation of China (Grant No.51667006).

\section{REFERENCES}

[1] M. Shen and Q. Gao, "A review on battery management system from the modeling efforts to its multiapplication and integration," Int. J. Energ. Res., pp. 2-33, 2019, doi: 10.1002/er.4433.

[2] E. Samadani, M. Mastali, S. Farhad, R.A. Fraser and M. Fowler, "Li-ion battery performance and degradation in electric vehicles under different usage scenarios," Int. J. Energ. Res., vol. 40, no. 3, pp. 379-392, 2016, doi: 10.1002/er.3378.

[3] C. Lyu, Q. Lai, T. Ge, H. Yu, L. Wang, and N. Ma, "A lead-acid battery's remaining useful life prediction by using electrochemical model in the Particle Filtering, framework," Energy, vol. 120, pp. 975-984, 2017, doi: 10.1016/j.energy.2016.12.004

[4] D. Liu, J. Zhou, D. Pan, Y. Peng, and X. Peng, "Lithium-ion battery remaining useful life estimation with an optimized Relevance Vector Machine algorithm with incremental learning," Measurement, vol. 63, pp. 143-151, 2015, doi: 10.1016/j.measurement.2014.11.031

[5] X. Hu, J. Jiang, D. Cao, and B. Egardt, "Battery Health Prognosis for Electric Vehicles Using Sample Entropy and Sparse Bayesian Predictive Modeling," IEEE Trans. Ind. Electron., vol. 63, pp. 2645-2656, 2016, doi: 10.1109/TIE.2015.2461523

[6] J. Wu, C. Zhang and Z. Chen, "An online method for lithium-ion battery remaining useful life estimation using importance sampling and neural networks," Appl. Energ., vol. 173, pp. 134-140, 2016, doi: 10.1016/j.apenergy.2016.04.057

[7] Y. Zhang, R. Xiong, H. He, and M. G. Pecht, "Long Short-Term Memory Recurrent Neural Network for Remaining Useful Life Prediction of Lithium-Ion Batteries," IEEE Trans. Veh. Technol., vol. 67, pp. 5695-5705, 2018, doi: 10.1109/TVT.2018.2805189

[8] L. Zhang, Z. Mu and C. Sun, "Remaining Useful Life Prediction for Lithium-Ion Batteries Based on Exponential Model and Particle Filter," IEEE Access, vol. 6, pp. 17729-17740, 2018, doi: 10.1109/ACCESS.2018.2816684

[9] Y. Xing, E. W. M. Ma, K. Tsui, and M. Pecht, "An ensemble model for predicting the remaining useful performance of lithium-ion batteries," Microelectron. Reliab., vol. 53, pp. 811-820, 2013, doi: 10.1016/j.microrel.2012.12.003

[10] Q. Miao, L. Xie, H. Cui, W. Liang, and M. Pecht, "Remaining u seful life prediction of lithium-ion battery with unscented particle filter technique," Microelectron. Reliab., vol. 53, pp. 805-810, 2013, doi: 10.1016/j.microrel.2012.12.004

[11] X. Su, S. Wang, M. Pecht, L. Zhao, and Z. Ye, "Interacting multiple model particle filter for prognostics of lithium-ion batteries," Microelectron. Reliab., vol. 70, pp. 59-69, 2017, doi: 10.1016/j.microrel.2017.02.003

[12] Y. Chang, H. Fang and Y. Zhang, "A new hybrid method for the prediction of the remaining useful life of a lithium-ion battery," Appl. Energ., vol. 206, pp. 1564-1578, 2017, doi: 10.1016/j.apenergy.2017.09.106

[13] J. L. Deng, "Control problems of grey systems," Syst. Control Lett., vol. 1, pp. 288-294, 1982, doi: 10.1016/S0167-6911(82)80025-X

[14] X. Li, Z. Wang, L. Zhang, C. Zou, and D. D. Dorrell, "State-of-health estimation for Li-ion batteries by combing the incremental capacity analysis method with grey relational analysis," J. Power Sources, vol. 410-411, pp. 106-114, 2019, doi: 10.1016/j.jpowsour.2018.10.069

[15] L. Chen, W. Lin, J. Li, B. Tian, and H. Pan, "Prediction of lithium-ion battery capacity with metabolic grey model," Energy, vol. 106, pp. 662-672, 2016, doi: 10.1016/j.energy.2016.03.096

[16] D. Q. Truong, K. K. Ahn and N. T. Trung, "Design of An Advanced Time Delay Measurement and A Smart Adaptive Unequal Interval Grey Predictor for Real-Time Nonlinear Control Systems," IEEE Trans. Ind. Electron., vol. 60, pp. 4574-4589, 2013, doi: 10.1109/TIE.2012.2213552

[17] S. Yin and X. Zhu, "Intelligent Particle Filter and Its Application on Fault Detection of Nonlinear System," IEEE Trans. Ind. Electron., vol.p. 1-1, doi: 10.1109/TIE.2015.2399396

[18] B. Saha and K. Goebel. "Battery Data Set", NASA Ames Prognostics Data Repository (http: //ti.arc.nasa.gov/tech/dash/groups/pcoe/prognostic-data-repository), NASA Ames Research Center, Moffett Field, CA, 2007. 
[19] L. Chen, Z. Lu, W. Lin, J. Li, and H. Pan, "A new state-of-health estimation method for lithium-ion batteries through the intrinsic relationship between ohmic internal resistance and capacity," Measurement, vol. 116, pp. 586-595, 2018, doi: 10.1016/j.measurement.2017.11.016

[20] L. Chen, Z. Wang, Z. Lu, J. Li, B. Ji, H. Wei, and H. Pan, "A Novel State-of-Charge Estimation Method of Lithium-Ion Batteries Combining the Grey Model and Genetic Algorithms," IEEE Trans. Power Electr., vol. 33, pp. 8797-8807, 2018, doi: 10.1109/TPEL.2017.2782721 


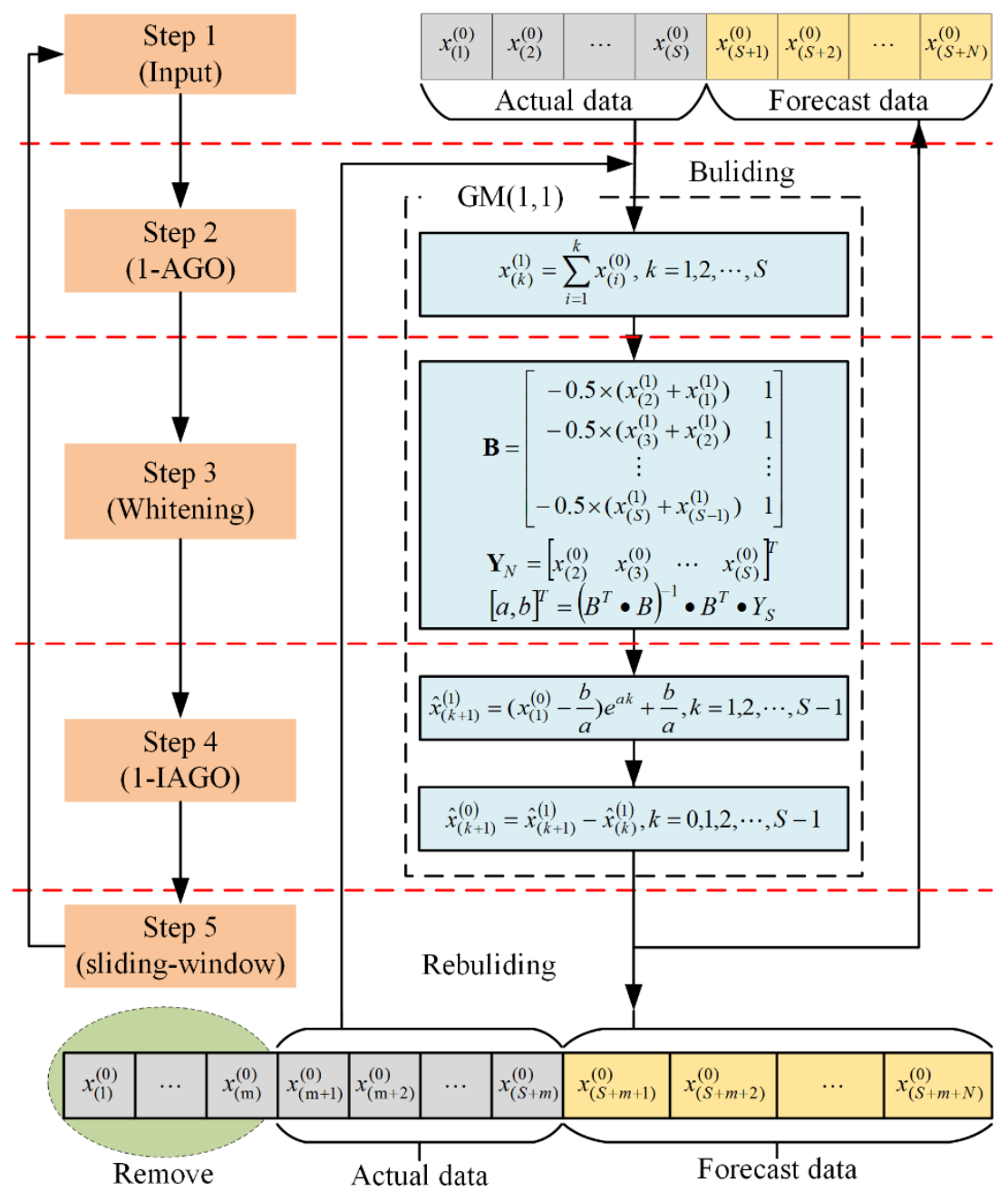

FIGURE. 1 Modeling procedure of SGM 


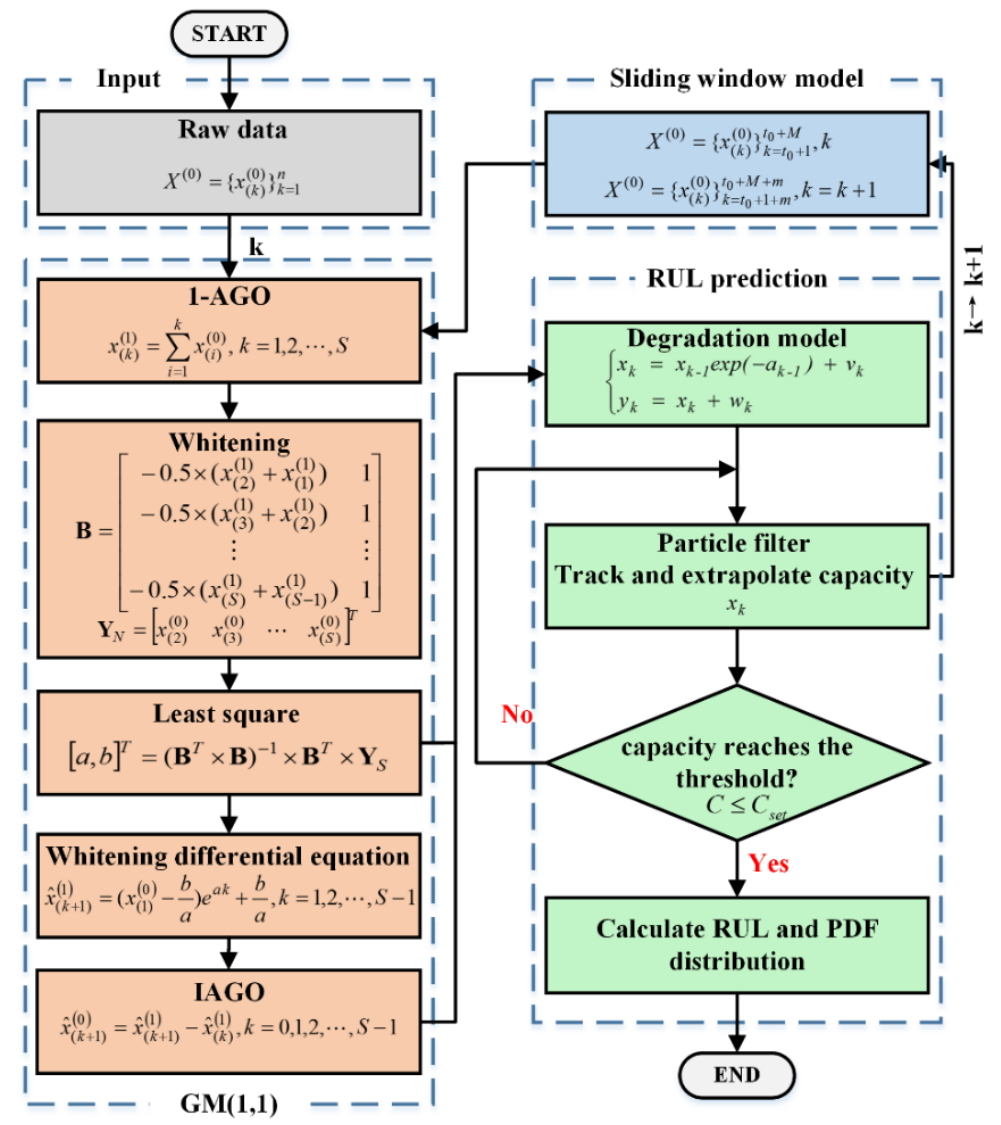

FIGURE. 2 The SGM-based PF framework for RUL prediction
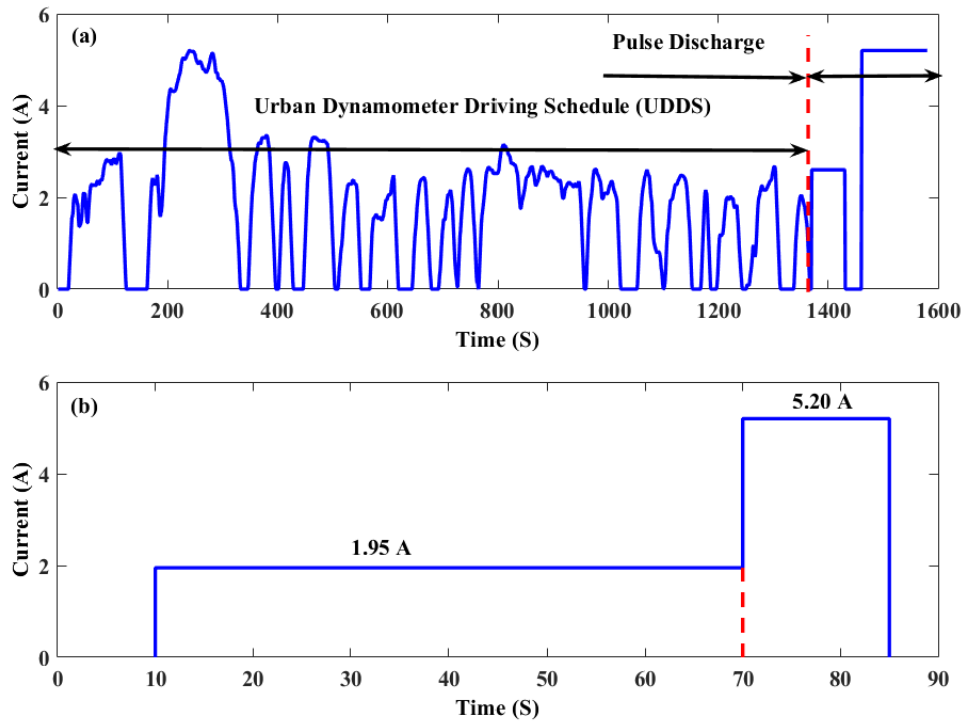

FIGURE. 3 Dynamic-current aging cycle test. (a) Urban Dynamometer Driving Schedule (UDDS) and Pulse Discharge. (b) Pulse Discharge 

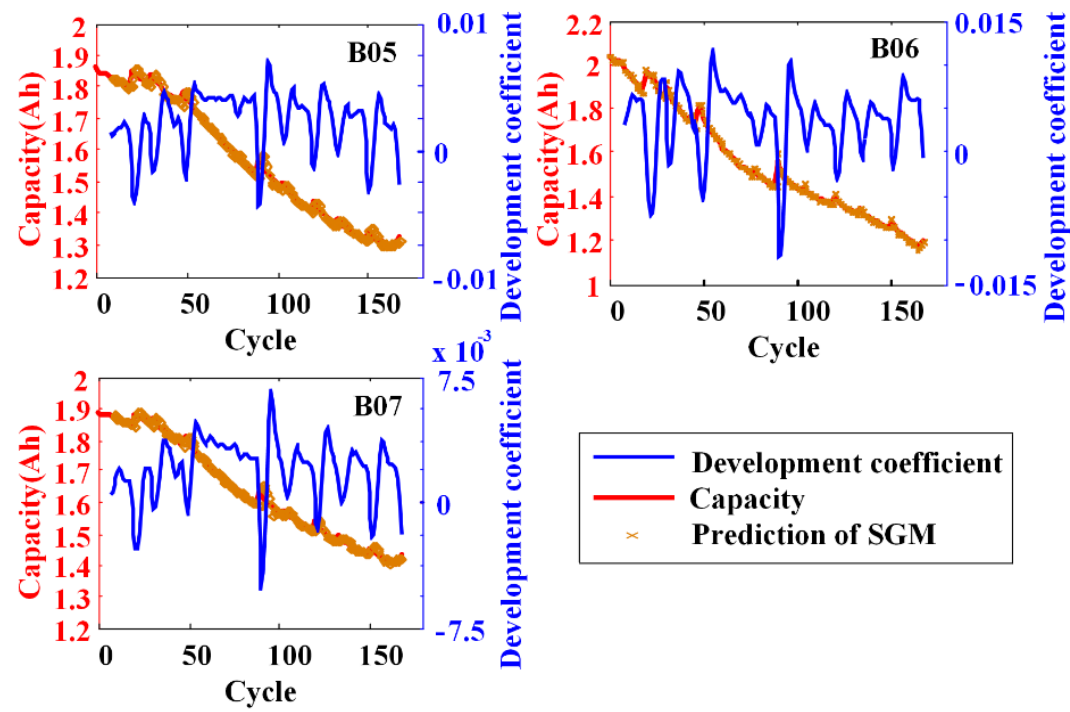

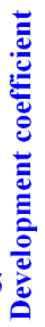

Development coefficient

Capacity

Prediction of SGM

FIGURE. 4 The capacity prediction results by SGM and the trajectories of development coefficient for B05, B06 and B07

(a)

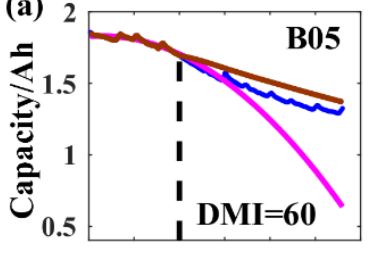

$\begin{array}{lllllllllll}0 & 30 & 60 & 90 & 120150180 & 0 & 40 & 80 & 120 & 160\end{array}$

(b)

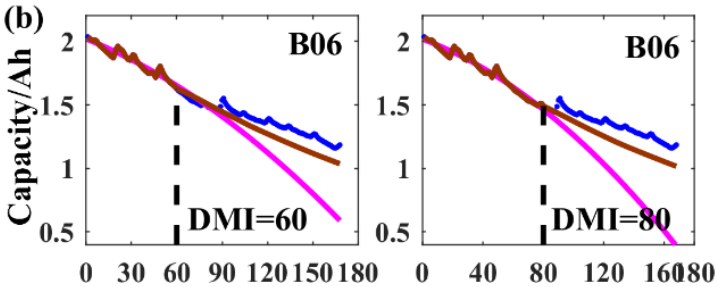

(c)

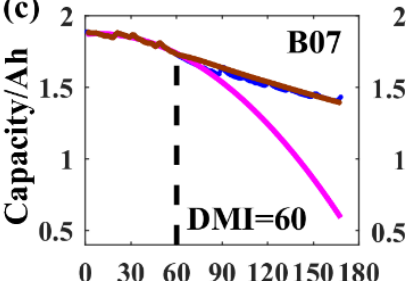

Cycle

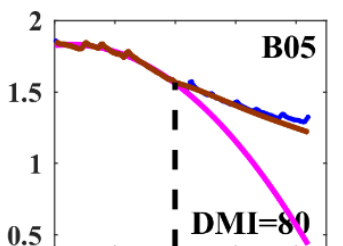

05
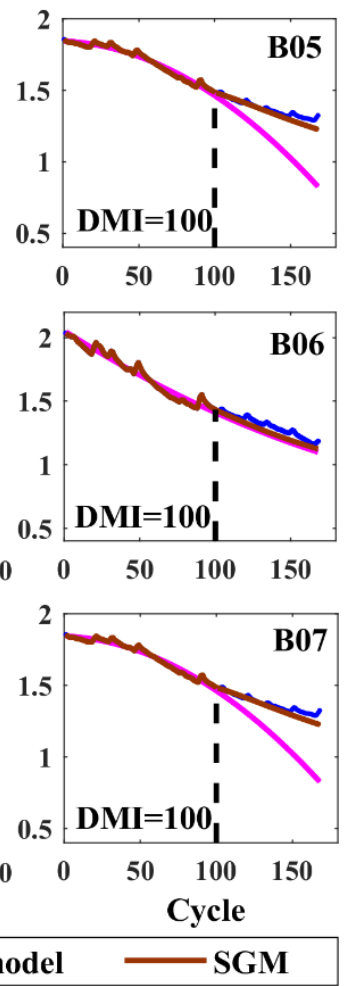

FIGURE. 5 The capacity prediction based on polynomial model and SGM at different DMI: (a) B05, (b) B06, and (c) B07 

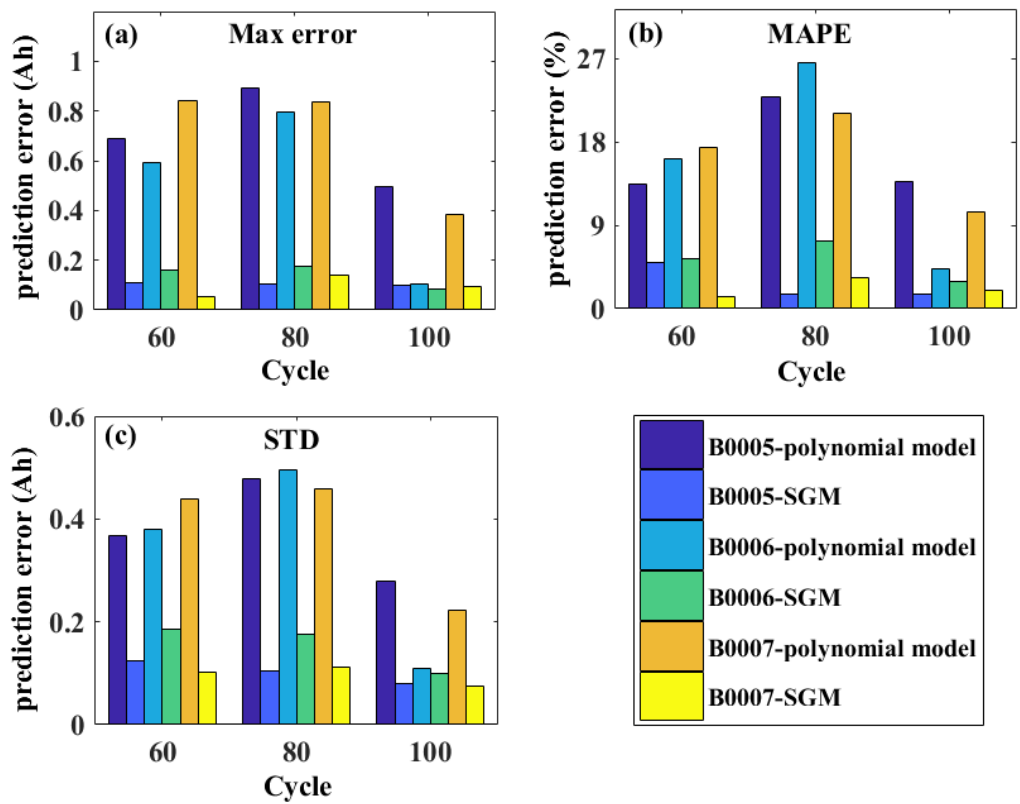

FIGURE. 6 The comparison of capacity prediction error of polynomial model and SGM: (a) Max errors; (b) MAPE; and (c) STD 

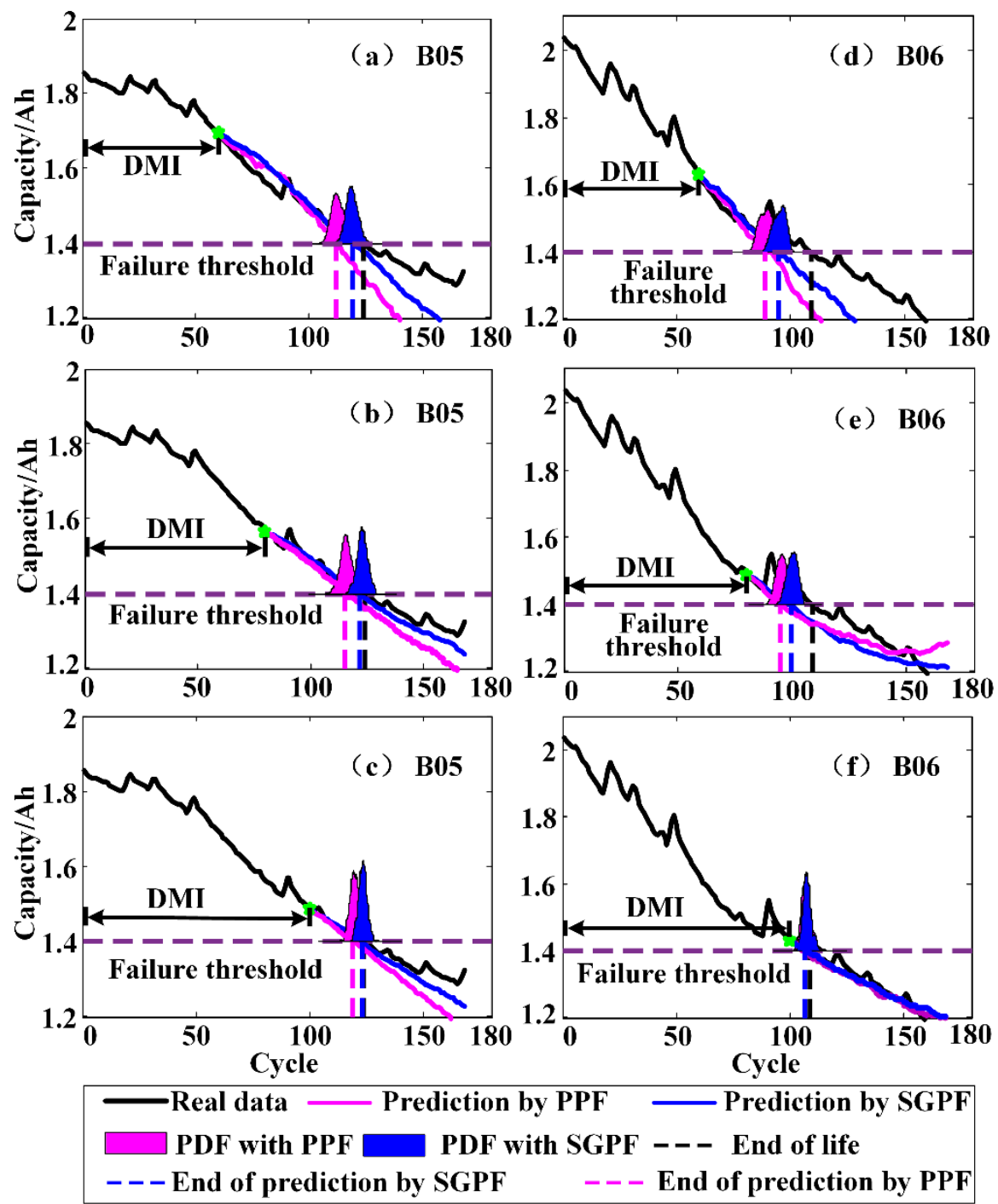

FIGURE. 7 RUL prediction for B05 and B06 using PPF and SGPF with different DMI 

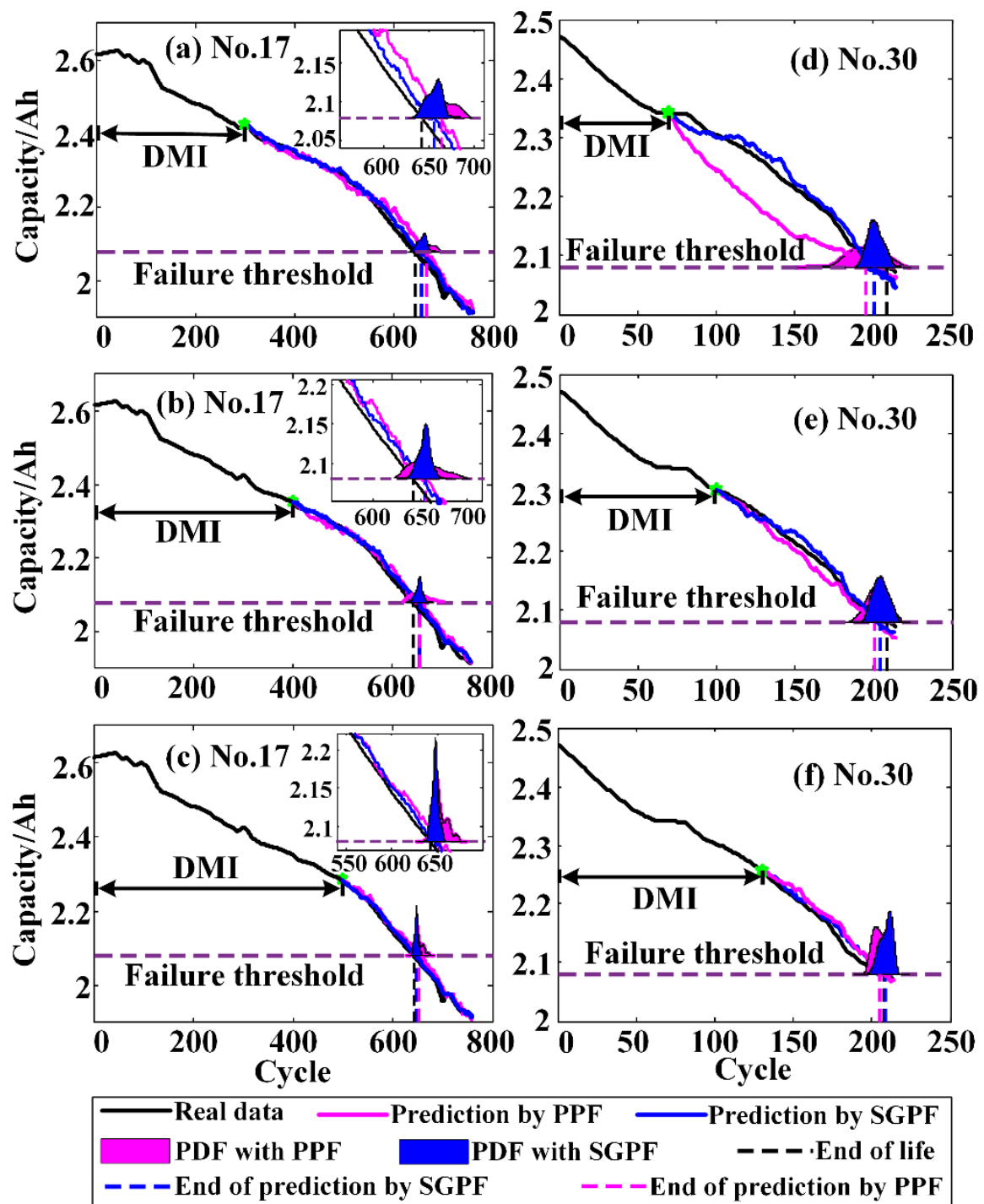

FIGURE. 8 RUL prediction for No. 17 and No. 30 using PPF and SGPF with different DMI 

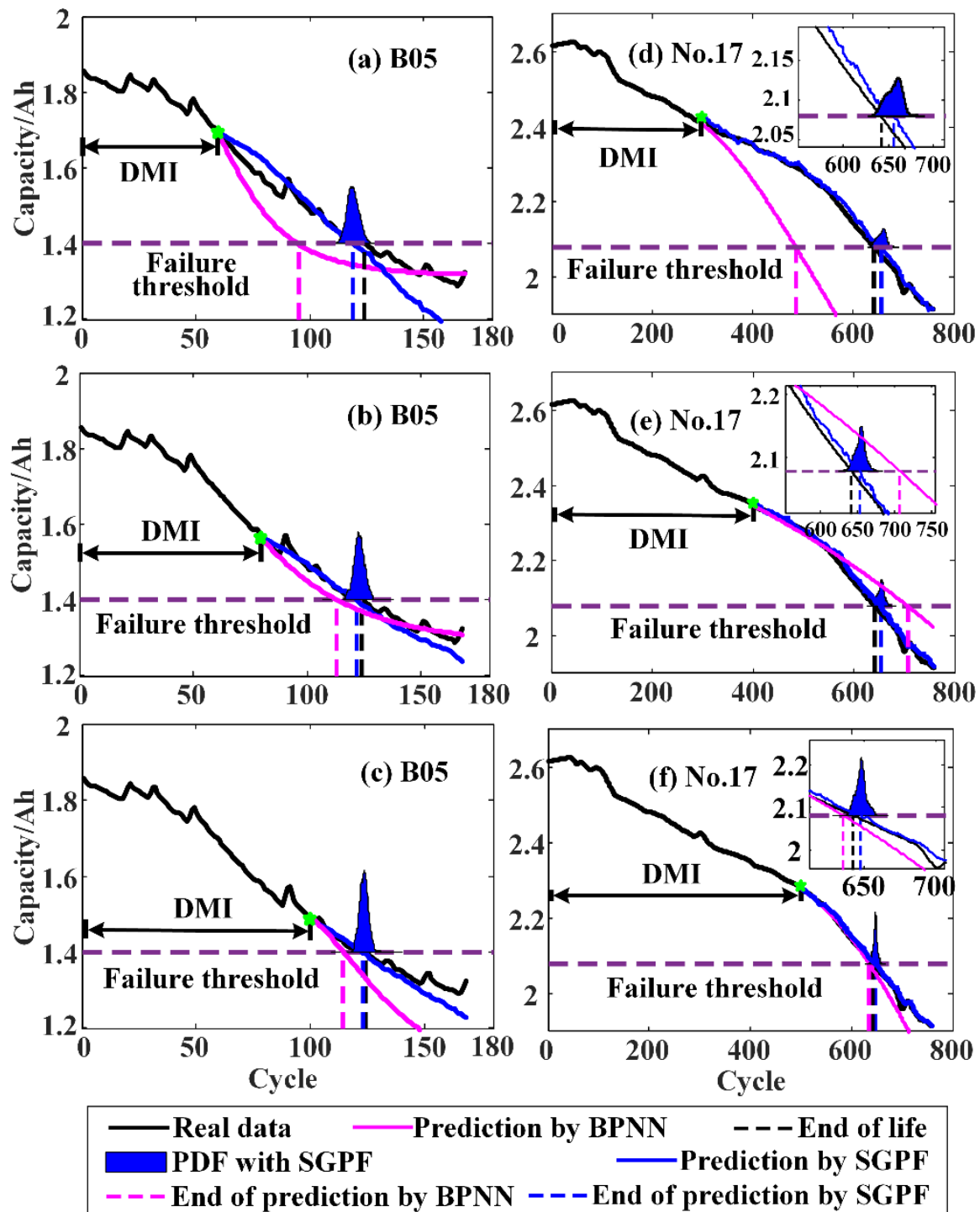

FIGURE. 9 RUL prediction for B05 and No.17 using BPNN and SGPF with different DMI 
Table 1 A comparison of the specifications of two types of batteries

\begin{tabular}{ccc}
\hline Battery & $\begin{array}{c}\text { Type 1 } \\
\text { Specifications }\end{array}$ & $\begin{array}{c}\text { Type 2 } \\
\text { (18650 Gen 2 from NASA) }\end{array}$ \\
\hline Rated capacity (Ah) & 2.0 & 2.6 \\
Nominal voltage (V) & $/$ & 3.7 \\
Charge cut-off Voltage (V) & 4.2 & 4.2 \\
Discharge cut-off voltage (V) & $2.7(\mathrm{~B} 05), 2.5(\mathrm{~B} 06)$ and 2.2 & 2.75 \\
Charging Method & (B07) & CC-CV \\
Charging Current & CC-CV & 1.3 \\
Fade in rated capacity (\%) & 1.5 & 20 \\
Aging conditions & 30 & Dynamic-current discharge \\
Voltage measurement accuracy & CC discharge & $\pm(0.05 \%+5 \mathrm{mV})$ \\
Current measurement accuracy & Not provided & $\pm(0.05 \%+10 \mathrm{~mA})$ \\
\hline
\end{tabular}

Table 2 The prediction results of capacity by SGM

\begin{tabular}{cccc}
\hline Battery & Max error $($ Ah $)$ & MAPE & STD (Ah) \\
\hline B05 & 0.0965 & $0.64 \%$ & 0.0129 \\
B06 & 0.1593 & $1.10 \%$ & 0.0244 \\
B07 & 0.1039 & $0.52 \%$ & 0.0117 \\
\hline
\end{tabular}

Table 3 Prediction results of three methods for five batteries with different DMI

\begin{tabular}{|c|c|c|c|c|c|c|c|c|c|c|c|c|c|c|c|c|c|}
\hline \multirow{2}{*}{ Sample } & \multirow{2}{*}{ EOL } & \multirow{2}{*}{ Method } & \multicolumn{5}{|c|}{$\mathrm{DMI}=1 / 3$ of whole life cycles } & \multicolumn{5}{|c|}{$\mathrm{DMI}=1 / 2$ of whole life cycles } & \multicolumn{5}{|c|}{ DMI $=2 / 3$ of whole life cycles } \\
\hline & & & RUL & $95 \% \mathrm{CI}$ & $\mathrm{AE}$ & AI & RMSE & RUL & $95 \% \mathrm{CI}$ & $\mathrm{AE}$ & AI & RMSE & RUL & $95 \% \mathrm{CI}$ & $\mathrm{AE}$ & AI & RMSE \\
\hline \multirow[t]{3}{*}{ B05 } & 124 & BPNN & 95 & 1 & 29 & $76.61 \%$ & 0.0682 & 113 & 1 & 11 & $91.13 \%$ & 0.0265 & 114 & 1 & 10 & $91.94 \%$ & 0.0224 \\
\hline & 124 & $\mathrm{PPF}$ & 112 & {$[108,118]$} & 12 & $90.32 \%$ & 0.0306 & 115 & {$[111,120]$} & 9 & $92.74 \%$ & 0.0168 & 119 & {$[116,124]$} & 5 & $95.97 \%$ & 0.0090 \\
\hline & 124 & SGPF & 119 & {$[115,124]$} & 5 & $95.97 \%$ & 0.0224 & 122 & {$[118,127]$} & 2 & $98.39 \%$ & 0.0091 & 123 & {$[120,127]$} & 1 & $99.19 \%$ & 0.0061 \\
\hline \multirow[t]{3}{*}{ B06 } & 109 & BPNN & 72 & 1 & 37 & $66.06 \%$ & 0.1424 & 89 & 1 & 20 & $81.65 \%$ & 0.2054 & 103 & 1 & 6 & $94.50 \%$ & 0.0202 \\
\hline & 109 & PPF & 89 & {$[84,96]$} & 22 & $79.82 \%$ & 0.0621 & 95 & {$[89,101]$} & 14 & $87.16 \%$ & 0.0369 & 107 & {$[102,114]$} & 2 & $98.17 \%$ & 0.0058 \\
\hline & 109 & SGPF & 95 & {$[90,99]$} & 14 & $87.16 \%$ & 0.0395 & 100 & {$[95,104]$} & 9 & $91.74 \%$ & 0.0297 & 107 & {$[104,110]$} & 2 & $98.17 \%$ & 0.0042 \\
\hline \multirow[t]{3}{*}{ В07 } & 165 & BPNN & 143 & 1 & 21 & $87.27 \%$ & 0.0440 & 155 & 1 & 9 & $94.55 \%$ & 0.0351 & 148 & 1 & 16 & $90.30 \%$ & 0.0334 \\
\hline & 165 & PPF & 147 & {$[141,153]$} & 18 & $89.09 \%$ & 0.0344 & 151 & {$[146,157]$} & 14 & $91.51 \%$ & 0.0304 & 156 & {$[150,162]$} & 9 & $94.55 \%$ & 0.0205 \\
\hline & 165 & SGPF & 151 & {$[147,155]$} & 14 & $91.51 \%$ & 0.0292 & 158 & {$[154,163]$} & 7 & $95.76 \%$ & 0.0212 & 160 & {$[157,164]$} & 5 & $96.97 \%$ & 0.0130 \\
\hline \multirow[t]{3}{*}{ No.17 } & 642 & BPNN & 486 & 1 & 156 & $75.86 \%$ & 0.1853 & 707 & 1 & 66 & $89.72 \%$ & 0.0216 & 635 & 1 & 6 & $99.07 \%$ & 0.0028 \\
\hline & 642 & $\mathrm{PPF}$ & 664 & {$[651,692]$} & 22 & $96.57 \%$ & 0.0175 & 655 & {$[630,695]$} & 13 & $97.98 \%$ & 0.0112 & 653 & {$[634,678]$} & 11 & $98.29 \%$ & 0.0090 \\
\hline & 642 & SGPF & 656 & {$[635,667]$} & 14 & $97.82 \%$ & 0.0090 & 654 & {$[645,663]$} & 12 & $98.13 \%$ & 0.0085 & 647 & {$[643,662]$} & 5 & $99.22 \%$ & 0.0056 \\
\hline \multirow[t]{3}{*}{ No.30 } & 209 & BPNN & 174 & 1 & 35 & $83.25 \%$ & 0.0571 & 200 & 1 & 9 & $95.69 \%$ & 0.0273 & 197 & 1 & 12 & $94.26 \%$ & 0.0140 \\
\hline & 209 & PPF & 195 & {$[175,211]$} & 14 & $93.30 \%$ & 0.0579 & 201 & {$[191,213]$} & 8 & $96.17 \%$ & 0.0119 & 205 & {$[198,212]$} & 4 & $98.09 \%$ & 0.0120 \\
\hline & 209 & SGPF & 201 & {$[193,208]$} & 8 & $96.17 \%$ & 0.0137 & 204 & {$[197,210]$} & 5 & $98.09 \%$ & 0.0109 & 209 & {$[204,213]$} & 0 & $100.00 \%$ & 0.0107 \\
\hline
\end{tabular}

